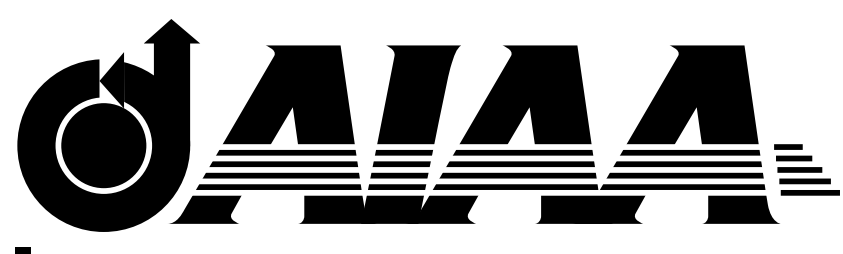

\title{
AIAA-2003-0082
}

\section{Zonal $k$-l based Large Eddy Simulations}

Paul G. Tucker

Fluid Dynamics Research Centre

The University of Warwick, Coventry, UK CV4 7AL

\section{Lars Davidson}

Department of Thermo and Fluid Dynamics

Chalmers University of Technology, SE-41296 Gothenburg, Sweden

\section{$41^{\text {st }}$ AIAA Aerospace Sciences Meeting and Exhibit}

\author{
6-9 January 2003 \\ Reno, Nevada
}

For permission to copy or republish, contact the copyright owner on the first page. For AIAA-held copy-right, write to AIAA, Permissions Department, 1801 Alexander Bell Drive, Suite 500, Reston, VA 20191-4344 


\title{
Zonal $k-/$ based Large Eddy Simulations
}

\author{
Paul G. Tucker* \\ Fluid Dynamics Research Centre \\ The University of Warwick \\ Coventry, CV4 7AL, UK \\ Lars Davidson** \\ Department of Thermo and Fluid Dynamics \\ Chalmers University of Technology \\ SE-41296 Gothenburg, Sweden
}

\section{ABSTRACT}

A straightforward way to implement zonal $k$-l based LES/RANS (Large Eddy Simulation/Reynolds Averaged Navier-Stokes) approach is presented. The interface location for the differing models is explicitly specified. Using standard established LES and RANS model constants the zonal method is shown to reproduce a satisfactory law of the wall. The approach is implemented in both cell-vertex and cell-centered codes. Channel flow predictions are found within about $3 \%$ of each other for the differing codes. Various other sensitivity studies are performed. These show that, as with standard LES, predictions are most sensitive to filter definition, first off wall grid node normal positions and temporal scheme order. For a periodic ribbed channel the new zonal LES approach heat transfer predictions are found to significantly more accurate than those for an established two equation RANS model.

\section{INTRODUCTION}

Relative to Unsteady Reynolds Averaged NavierStokes (URANS) separated flow solutions (see Tucker $^{1}$ ), LES (Large Eddy Simulation) is especially promising. However, it is still computationally expensive. Also, in wall regions, LES presents difficulties $^{2}$. According to Spalart ${ }^{3}$, due to expense, LES is unlikely to see use in aircraft design until around the year 2045. Therefore, Spalart proposes the use of Detached Eddy Simulations (DES). With these, the RANS equations are just solved adjacent to solid surfaces. In areas where there are detached unsteady eddies LES is applied. Hence DES could be viewed as a zonal or hybrid technique. Shur et al. ${ }^{4}$ apply DES to flow over a high incidence wing, hence having unsteady vortex shedding). Niktin et al. ${ }^{5}$

\footnotetext{
* Senior Lecturer in Fluid Mechanics

${ }^{* *}$ Head of Department of Thermo and Fluid Dynamics
}

perform plane channel flow DES simulations, Forsythe et al. ${ }^{6}$ axisymmetric base flow, and Kumar and Loth $^{7}$ iced-wing simulations. In all these the Spalart and Allmaras ${ }^{8}$ turbulence model is adapted for both the LES and RANS regions and promising results found. Importantly, the RANS/LES interface is at $y \approx C \Delta$ where $y$ is nearest wall distance, generally $C=0.65$ and $\Delta$ is a filter (grid) width. The latter is based on the maximum cell extent. Therefore, grid structure influences the LES/RANS interface location.

Along different lines, Speziale ${ }^{9}$ implies zonal LES can be implemented through scaling RANS modelled turbulent stresses by the function $\left[1-\exp \left(-C \Delta / l_{k}\right)\right]^{n}$ where $C$ and $n$ are undetermined constants and $l_{k}$ is the Kolmogorov length scale. As the grid becomes finer (i.e. $\Delta$ smaller) the scaling function and hence modelled stress tends to zero. Therefore, for sufficiently fine grids Direct Numerical Simulation (DNS) will occur. Conversely for large $\Delta$ values, RANS modelling will take place. Mostly of a preliminary nature, zonal/hybrid approaches, inspired by Speziale's attractive idea, are presented by Arunajatesan, Sinha and Menon ${ }^{10}$, Batten, Goldberg and Chakravarthy ${ }^{11}$, Peltier, Zajaczkowski and Wyngaard $^{12}$, and Arunajatesan and Sinha ${ }^{13}$. As part of the listed work, both plane and ribbed channel predictions are made. A key aspect of the Speziale approach is the accurate determination of $C$ and $n$ which indirectly control the LES/RANS interface location.

Davidson ${ }^{14}$ explores a third zonal approach. With this, the LES/RANS interface is rigidly fixed in the logarithmic boundary layer region at a specified dimensionless wall distance $\left(y^{+}\right)$. A $k-l$ subgrid scale model is used with a $k-\omega$ near wall RANS approach. Although preliminary results are promising, the introduction of two turbulence models is to an extent undesirable adding model complexity (especially at the model interface matching line). Therefore, here, 
this work is revised, using a $k$-l based model through the complete domain. Initially, channel flow simulations are performed. Then the new hybrid $k-l$ approach is applied to a separated, non-isothermal ribbed channel flow. It should be noted that rigid enforcement of the LES/RANS interface will generally give rise to non-smooth turbulence variables. One remedy is (for simple geometries) careful grid design. As with the method of ${ }^{3}$ grid exerts a strong solution control. Another remedy is, reminiscent of method of Spalart ${ }^{3}$, to set the turbulence length scale as a function that takes the minimum of the RANS and LES scales. In a k-l framework this will tend to bring the zonal modelmatching interface in significantly closer to walls than in the method of Spalart ${ }^{3}$. In the present work, just fixing the RANS/LES interface at a distance that is further out than the 'natural' interface is explored.

\section{NUMERICAL METHOD}

\section{General governing equations}

Conservation of momentum

$$
\begin{aligned}
\frac{\partial \tilde{u}_{i}}{\partial t}+\frac{\partial \tilde{u}_{i} \tilde{u}_{j}}{\partial x_{j}} & =\frac{\delta_{1 j} \beta}{\rho}-\frac{1}{\rho} \frac{\partial \tilde{p}}{\partial x_{i}}+ \\
& \frac{1}{\rho} \frac{\partial}{\partial x_{j}}\left[\left(\mu+\mu_{T}\right)\left(\frac{\partial \tilde{u}_{i}}{\partial x_{j}}\right)\right]
\end{aligned}
$$

and energy

$$
\begin{aligned}
& \frac{\partial \tilde{T}}{\partial t}+\frac{\partial \tilde{u}_{i} \tilde{T}}{\partial x_{j}}= \frac{\delta_{1 j} \alpha \tilde{u}_{1}}{\rho}+ \\
& \frac{1}{\rho} \frac{\partial}{\partial x_{j}}\left[\left(\frac{\mu}{\operatorname{Pr}}+\frac{\mu_{T}}{\operatorname{Pr}_{T}}\right)\left(\frac{\partial \tilde{T}}{\partial x_{j}}\right)\right]
\end{aligned}
$$

can be expressed using the above two equations, respectively. The symbol $\tilde{u}_{i}$ is a fluid velocity component, $\tilde{T}$, reduced temperature, $\rho$ density, $\mu$ viscosity, $\tilde{p}$ static reduced pressure, $t$ time and $x$ the spatial co-ordinate. Following Peltier et al. ${ }^{12}$, the tilda is used to identify that near walls traditional RANS time averaging is applied and that elsewhere LES space filtering is implemented. Consequently, in the RANS region $\mu_{T}=\mu_{t}$, the eddy viscosity. For the LES region $\mu_{T}=\mu_{\text {sgs }}$, the subgrid scale viscosity.
Correspondingly, for the LES and RANS regions $\operatorname{Pr}_{T}$ $=0.4$ and 0.9 , respectively. The parameters $\alpha$ and $\beta$ are mean temperature and pressure gradients, respectively. These aid implementation of the periodic streamwise boundary conditions (see Patankar et al. $^{15}$ ). The continuity equation corresponding to the above is

$$
\frac{\partial \tilde{u}_{j}}{\partial x_{j}}=0
$$

\section{Zonal turbulence model details}

The modelled turbulent kinetic energy, $k$, equation is as follows

$$
\begin{aligned}
& \frac{\partial k_{T}}{\partial t}+\frac{\partial \tilde{u}_{i} k_{T}}{\partial x_{j}}= \\
& \quad \frac{1}{\rho} \frac{\partial}{\partial x_{j}}\left[\left(\mu+\frac{\mu_{T}}{\sigma_{k}}\right)\left(\frac{\partial k_{T}}{\partial x_{j}}\right)\right]+P_{k_{T}}-\varepsilon_{T}
\end{aligned}
$$

where $\sigma_{k}=1$ is the diffusion Prandtl number for $k$ and $P_{k_{T}}$ the turbulence production term. Again the ' $T$ subscript is used to differentiate between subgrid and Lagrangian related components.

For the RANS regions, the $k-l$ models of Wolfshtein ${ }^{16}$, and Chen and Patel ${ }^{17}$ are tried. In the LES region, the subgrid $k_{\text {sgs }}-I$ model of Yoshizawa ${ }^{18}$ and Fureby ${ }^{19}$ are used. For all the above models Equation (4) is solved with $\varepsilon_{T}=C_{\varepsilon} k_{T}^{3 / 2} / l_{\varepsilon}$ and $\mu_{T}=\rho C_{\mu} l_{\mu} k^{1 / 2}$. To incorporate the $k_{\text {sgs }}-1$ model the $C_{\varepsilon}$ constant has been included. Also, length scales are modified as follows

$$
l_{\mathcal{\varepsilon}}=n C_{\varepsilon 2} y\left(1-e^{-A_{\mathcal{E}} y^{*}}\right)+(1-n) \Delta
$$

and

$$
l_{\mu}=n C_{\mu 2} y\left(1-e^{-A_{\mu} y^{*}}\right)+(1-n) \Delta
$$

where $y^{*}=y \rho k_{T}^{1 / 2} / \mu, \Delta$ is a filter width and $n=1$ or 0 activates RANS and LES modelling, respectively. The following possible $\Delta$ forms are considered: $\Delta=$ $\Delta y\left(\right.$ Davidson $\left.^{14}\right), \Delta=\Delta x\left(\right.$ Niktin et al. $\left.{ }^{5}\right), \Delta=(\Delta x \Delta y$ 
$\Delta z)^{1 / 3}$ (Peltier et al. $^{12}$ ). Model parameters that naturally admit the RANS and LES models are given in Table 1. At the RANS/LES interface, there is no guarantee that the turbulence length scale will be continuous. Therefore, smoothing is desirable. Both Codes (A) and (B) have multigrid convergence acceleration. The restriction operators in these give ready-made smoothing subroutines. For the fully developed (two-dimensional geometry) channel flow prediction, the 27-point, three-dimensional, restriction operator essentially reduces to the following onedimensional expression for a smoothed length scale $I_{s}$

$$
l_{s}^{\text {new }}=\frac{l_{j+1}^{\text {old }} \delta y_{j-1}+l_{j}^{\text {old }}\left(\delta y_{j+1}+\delta y_{j-1}\right)+l_{j-1}^{\text {old }} \delta y_{j+1}}{2\left(\delta y_{j+1}+\delta y_{j-1}\right)}
$$

\begin{tabular}{|l|l|l|l|l|l|l|l|}
\hline Model & $n$ & $A_{\varepsilon}$ & $A_{\mu}$ & $C_{\varepsilon 1}$ & $C_{\varepsilon 2}$ & $C_{\mu 2}$ & $C_{\mu}$ \\
\hline Wolfshtein & 1 & 0.263 & 0.016 & 1 & 2.400 & 2.400 & 0.09 \\
\hline $\begin{array}{l}\text { Chen \& } \\
\text { Patel }\end{array}$ & 1 & 0.200 & 0.014 & 1 & 2.495 & 2.495 & 0.09 \\
\hline $\begin{array}{l}\text { Yoshizawa/ } \\
\text { Fureby }\end{array}$ & 0 & - & - & 1.05 & - & - & 0.07 \\
\hline
\end{tabular}

Table 1 Turbulence modelling constants

where $\delta y$ represent vertical grid node spacings on a $j$ $1, j, j+1$ finite difference stencil and the 'new' and 'old' superscripts are now used in an iterative sense. Equation (7) can be applied several times to $I$. The number of applications increases the smoothing level. Here it is applied three times after each length scale re-evaluation. The RANS model needs $y$. To avoid discontinuities, for the ribbed channel this is obtained by solving the following elliptic differential equation

$$
{\frac{\partial}{\partial x_{j}}}_{j}\left(\frac{\partial L}{\partial x_{j}}\right)=C
$$

where $C=-1$, and at solid walls $L=0$; otherwise the condition $\partial L / \partial x=0$ is used. Here, $y$ is related to $L$ using the negative root of the expression below

$$
y=-\left|\frac{\partial L}{\partial x_{j}}\right| \pm \sqrt{\left(\left(\left|\frac{\partial L}{\partial x_{j}}\right|\right)^{2}+2 L\right)}
$$

More details of the above differential equation wall distance approach can be found in Tucker ${ }^{1}$. For the channel flow case $y$ is algebraically evaluated.

\section{General solution techniques}

As part of the work, comparison is made between plane channel flow solutions using two different codes. Code $(A)$ uses the less common cell-vertex scheme. With this, control volume fluxes are well approximated. However, for stretched grids, variables are not stored at the cell centres. Hence, the stored variable is not always such a good representation of values over the complete volume. Code $B$ uses the more popular approach of storing variables at control volume centres. For this, the error traits are reversed, control volume face flux values being less well modelled. A further key difference is that Codes (A) and $(B)$ use staggered and collocated variables, respectively. Other factors that should be born in mind is that Code $(A)$ uses second order backwards differences at walls, and is strictly Cartesian and Code (B) allows for body fitted grids. Clearly, with sufficiently fine grids, code differences should vanish. However, the nature of the present work is that URANS based limitations are placed on grid refinement. Hence, some slight numerical differences are to an extent expected. Codes $(A)$ and $(B)$ both have the option of SIMPLE based or fractional step pressure prediction procedures. Full numerical details for codes (A) and (B) can be found in Tucker ${ }^{1}$, and Davidson $^{14}$ or Sohankar and Davidson ${ }^{20}$, respectively. A Code (A) CDROM can be found with Tucker (2001). Verification of the Code (A) temporal accuracy, by comparing predictions with an eigen solution of the Orr-Sommerfeld equation for a travelling Tollmien-Schlichting wave, can be found in Chung and Tucker ${ }^{21}$. For all predictions presented here, the codes use $2^{\text {nd }}$ order central differences, with the Crank-Nicholson temporal scheme for both the convection and diffusion terms. For the nonisothermal channel flow, since $\operatorname{Pr}_{T}$ is subjected to a step change, harmonic means are used to evaluate discretized temperature equation diffusion coefficients. 


\section{Plane channel flow simulation details}

For plane channel simulations $R e_{\tau}=1050$. The $(x, y, z)$ domain size of $2 \pi \times 2 \times \pi$ is discretized with a $33 \times 65 \times 33$ grid involving a cross-stream $(y)$ geometric grid expansion factor of 1.15. This factor ensures $y^{+}$values at $1^{\text {st }}$ off-wall nodes $\left(y^{+} f\right)$ are around unity. The grid is such that $\Delta x^{+} \approx 200$ and $\Delta z^{+}$ $\approx 100$. The latter spacing is too big for an accurate LES. Hence, near wall RANS modelling is needed. For channel predictions the LES/RANS model interface $\left(y_{1}^{+}\right)$is placed at $y^{+}=60$. For the ribbed channel case, as discussed in the next section, the LES/RANS interface location aspect is more complex.

\section{Ribbed channel simulation details}

For the periodic ribbed channel, Code $(A)$ is used. Comparison is made with the measurements of Rau et al. ${ }^{22}$ having $R e=30,000$ (based on the channel height $y_{\text {max }}$ ). The adiabatic rib pitch is nine times the rib height. The walls have a constant heat flux. The mean flow driving pressure gradient is set to maintain the measured mass flow rate, $\dot{m}_{o}$. Initially the following expression ${ }^{23}$ is used

$$
\begin{aligned}
\delta_{1 j} \beta=\frac{\partial p^{\text {new }}}{\partial x} & =\frac{\partial p^{\text {old }}}{\partial x} \\
& -\frac{\left(\dot{m}^{\text {new }}-\dot{m}_{o}\right)-1 / 2\left(\dot{m}^{\text {old }}-\dot{m}_{o}\right)}{1 / 2 \Delta t z_{\max } y_{\max }}
\end{aligned}
$$

where the superscripts 'old' and 'new' represent time levels and the subscript 'max' maximum values. However, since Equation (10) did not suggest a constant pressure gradient, final solutions are based on a fixed $\beta$. This value is based on previous time varying values.

The average shear stress for the upper wall is significantly higher than for the separated lower wall flow. Since, the focus of the present work is the rib, it is decided to optimize the grid with respect to this geometric feature. If the upper and lower walls were both of importance, a considerably more complex grid design is required. Such issues are discussed by Spalart ${ }^{24}$. Simulations use a $103 \times 102 \times 33(x, y, z)$ grid. To ensure $y_{f}^{+} \approx 1$, in $x$ and $y$ a stretched grid is used with a maximum expansion factor of 1.25.
Based on the lower wall average shear stress (since $y^{+}=y \sqrt{\rho \tau_{w}} / \mu$ ) the following approximate lower wall expression is gained $y^{+} \approx C y$ where $C$ $=\sqrt{\rho \tau_{w}} / \mu \approx 2.5 \times 10^{4}$. Considering this relationship and assuming that the matching region extent should be less than $20 \%$ of the rib size suggests $y^{+}, \approx 30$. This $y_{1}^{+} \approx 30$ criterion is enforced by ensuring $n=1$ (see equations (5) and (6)), $\operatorname{Pr}_{T}=0.4$ for $y<y^{+}, / C$ and $n=0, \operatorname{Pr}_{T}=0.9$ for $y \geq y^{+}, / C$. Setting the matching region extent on wall distance (rather than grid node numbers) based intrinsic arguments is more convenient for complex geometries. This is especially the case when a differential equation based wall distance equation is implemented as with Code (A) (see later).

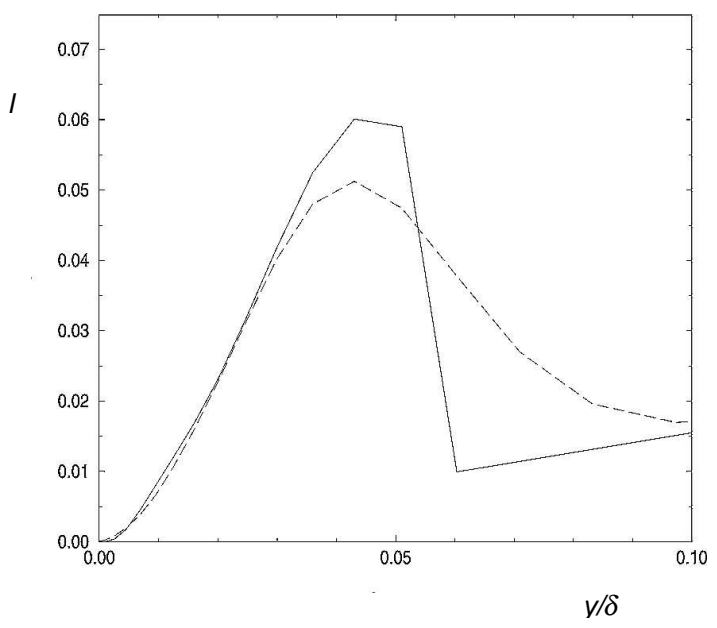

Fig. 1. Smoothing effect of Equation (7) (Smoothed length scale $\left(I_{s}\right)$, --.- Non-smooth scale $(I)$ )

\begin{tabular}{|l|l|}
\hline Model & $\%$ Change \\
\hline Different code & +3.2 \\
\hline$C P_{-} Y F$ constants & +0.99 \\
\hline Cross term omission & +1.15 \\
\hline$\Delta=(\Delta x \Delta y \Delta z)^{1 / 3}$ & +8.7 \\
\hline$\Delta=\Delta y$ & +12.4 \\
\hline$y^{+}=30$ & +2.9 \\
\hline$y_{f}^{+}=3$ & -15 \\
\hline No / smoothing & +2.2 \\
\hline $1^{\text {st }}$ order temporal scheme & 11.8 \\
\hline
\end{tabular}

Table 2 Sensitivity studies 


\section{RESULTS AND DISCUSSION}

\section{Plane Channel Flow Results}

Fig. 1 shows Equation (7) smoothing effect on $l_{\mu}$ after three passes. As can be seen, pleasingly smoothing is localized around the region of most importance $(y / \delta=0.4)$ (please note Fig. 1 uses $\Delta=\Delta y$ which gives by far the biggest length scale mismatch, $\Delta=(\Delta x \Delta y$ $\Delta z)^{1 / 3}$ length scales are much smoother). Table 2 suggests approximate model parameter and code sensitivities. These are characterized by centre line velocity changes. They are generally considered relative to a datum Code (A) solution with Wolfshtein $^{16}$, Yoshizawa $^{18}$, Fureby ${ }^{19}$ (W_YF) constants, $\Delta=\Delta y, y_{l}^{+}=60$ and $y_{f}^{+}=1$. Also, for the datum prediction, cross terms are included in the Navier-Stokes equations and turbulence length scales are smoothed at the matching interface. The datum prediction's centre line velocity is $8.5 \%$ too low (this is because, in a more challenging fashion, the pressure gradient rather than $\dot{m}$ is fixed) and + or - signs are used to indicate accuracy improvements and losses, respectively. In the first table entry, indicating the change to Code (B) sensitivity study, there is no length scale smoothing (the comparison accounts for this). For the second table entry, the Wolfhshtein RANS constants are replaced by those of Chen and Patel ${ }^{17}$ giving what are designated $C P \_Y F$ constants.

The table suggests that for accuracy $y_{f}^{+} \leq 1$ is important. It also correctly indicates that using a $1^{\text {st }}$ order temporal scheme (with a $2^{\text {nd }}$ order scheme suited $\Delta t$ ) makes a significant difference to results. Numerical studies show that to gain similar results to those for the $2^{\text {nd }}$ order temporal scheme, much smaller first order time steps are required. Hence, $1^{\text {st }}$ order temporal scheme performance is poor for the present flow. Pleasingly, results show relatively low sensitivity to turbulence model constants.

Fig. 2 gives law of the wall comparisons with the 'benchmark' LES data of Piomellit $i^{25}$ denoted using symbols. The dotted and dashed lines are for Codes (B) and (A), respectively, with $C P_{-} Y F$ constants, nocross terms, $\Delta=\Delta y$ and $y^{+}{ }_{l}=\overline{60}, y_{f}^{+}=1$ and no smoothing. As can be seen, the Code (B) results are closer to the LES data. However, the around $3 \%$ difference in results between the codes can probably be attributed to the quite different numerical discretizations noted earlier. Plots of turbulence normal stress parameters show similar relatively small discrepancy levels. The Fig. 2 full line gives Code (A) predictions with $W_{-} Y F$ constants, smoothing, cross-terms, $y^{+}{ }_{l}=60, y^{+}{ }_{f}^{-}=1$ and $\Delta=(\Delta x$
$\Delta y \Delta z)^{1 / 3}$. As shown in Table 2, the latter factor (the filter choice) has one of the stronger solution influences. This key change has resulted in reasonable law of the wall agreement. Since: the volume weighted filter scale is more applicable to the ribbed channel case; smoothing to avoid discontinuous solution variables seems numerically important and agreement is reasonable, the full line Code (A) solution parameters are applied to the

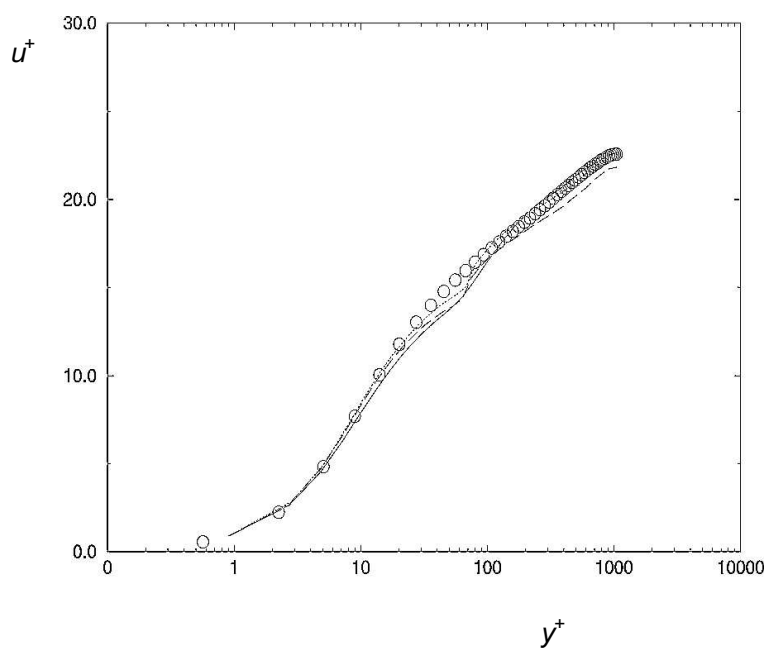

Fig. 2. Law of the wall comparisons with the 'benchmark' data (o 'benchmark' data; '..... Code (B), $\Delta=\Delta y$; --- Code (A), $\Delta=\Delta y$; - Code (A), $\Delta=$ $\left.(\Delta x \Delta y \Delta z)^{1 / 3}\right)$

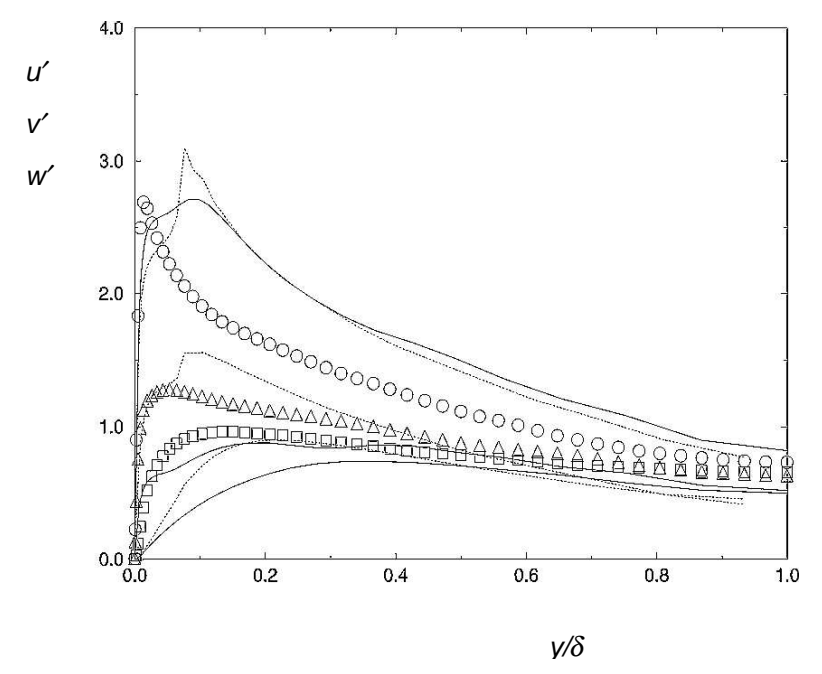

Fig. 3. Plane channel flow turbulent stress components (o 'benchmark' data; '.... Code (B), $\Delta=\Delta y$; - Code (A), $\left.\Delta=(\Delta x \Delta y \Delta z)^{1 / 3}\right)$ 
ribbed channel. However, before this, some turbulence stress component plots are considered in Fig. 3.

These relate to the Fig. 2 Code $(B)$ results (dotted line) and full line (volume based filter scale) Code (A) results. The line styles for Fig. 3 are kept the same. Again, symbols are used for the LES data. The combined effects of having different codes, constants, filters, smoothing levels, and solutions with and without cross terms has given rise to clear results differences. Fig. 3 shows smoothing has made cosmetic improvements. Generally, the Code

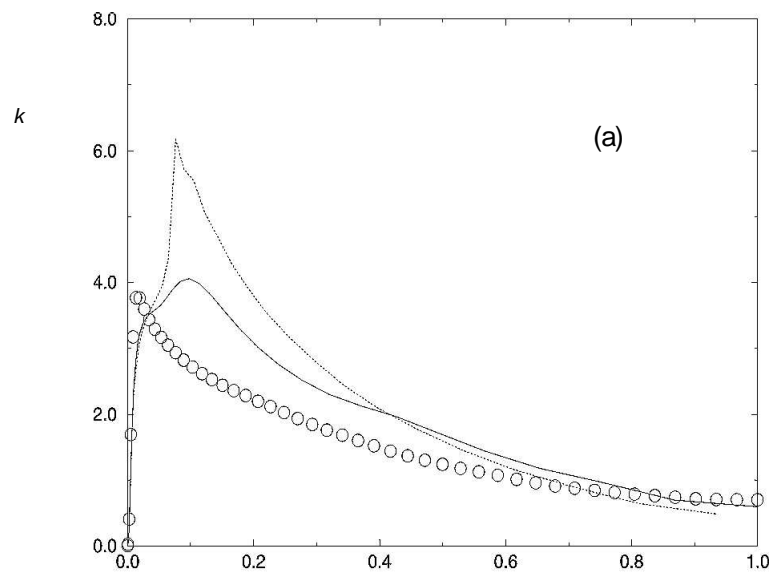

$y / \delta$

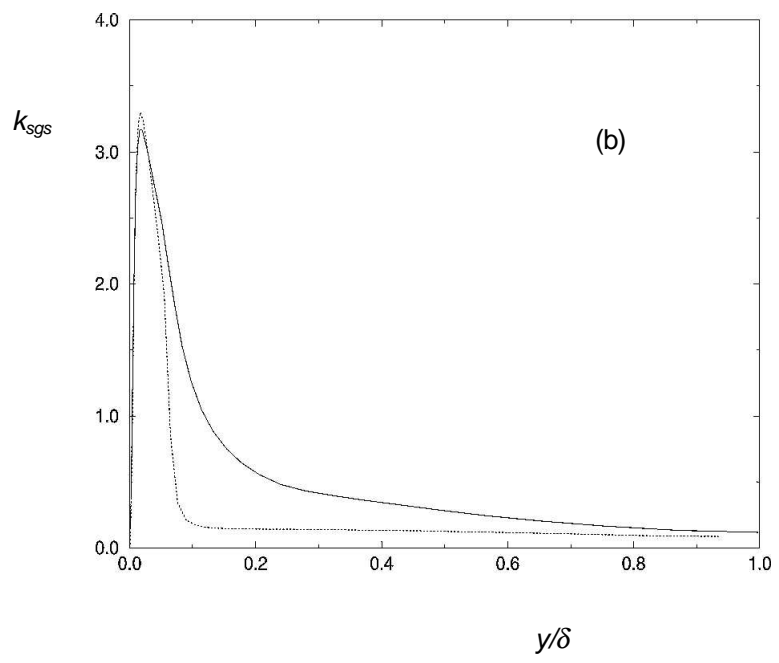

Fig. 4. Variation of resolved and modelled kinetic energy with $y / \delta$ : (a) resolved and (b) modelled. (o 'benchmark' data; Code (B), $\Delta=\Delta y$; - Code (A), $\left.\Delta=(\Delta x \Delta y \Delta z)^{1 / 3}\right)$

(A) stress components are lower than those for (B). This point is highlighted in Fig. 4a, plotting the resolved turbulence kinetic energy with $y$. Fig. 4b gives the modelled $k$ components. In a consistent fashion with Fig. 4a, Code $(A)$ results have a greater modelled element. Since the Code (A) length scale

definition also involves the larger non-normal grid spacings this is to be expected. The greater modelled element aspect is further reflected in the Fig. $5 \mu / \mu_{T}$ plots.

\section{Ribbed channel simulation details}

Fig. 6, compares predictions with the measur-ements of Rau et al. ${ }^{22}$ for Nusselt number $(\mathrm{Nu})$ along the bottom channel wall between the ribs. The $x$ coordinate is normalized by the rib height to give $X$ and $N u$ by the Nusselt number for a channel with no ribs $\left(N u_{0}\right)$. The latter is evaluated from the Dittus-

Boelter equation $\left(\mathrm{Nu}_{0}=0.023 \operatorname{Re}^{0.8} \mathrm{Pr}^{0.4}\right)$. The full line represents the current zonal LES simulations. The dashed gives results for the well-known Launder and Sharma ${ }^{26}$ low Reynolds number $k-\varepsilon$ model. As can be seen, the current zonal LES results are most accurate. The zonal predictions are also more accurate than Code (A) pure LES (not shown on the graph) by about $5 \%$. The pure LES tends to overpredict $\mathrm{Nu}$. Mean velocity and turbulence intensity comparisons with measurements also suggest good relative zonal LES model performance.

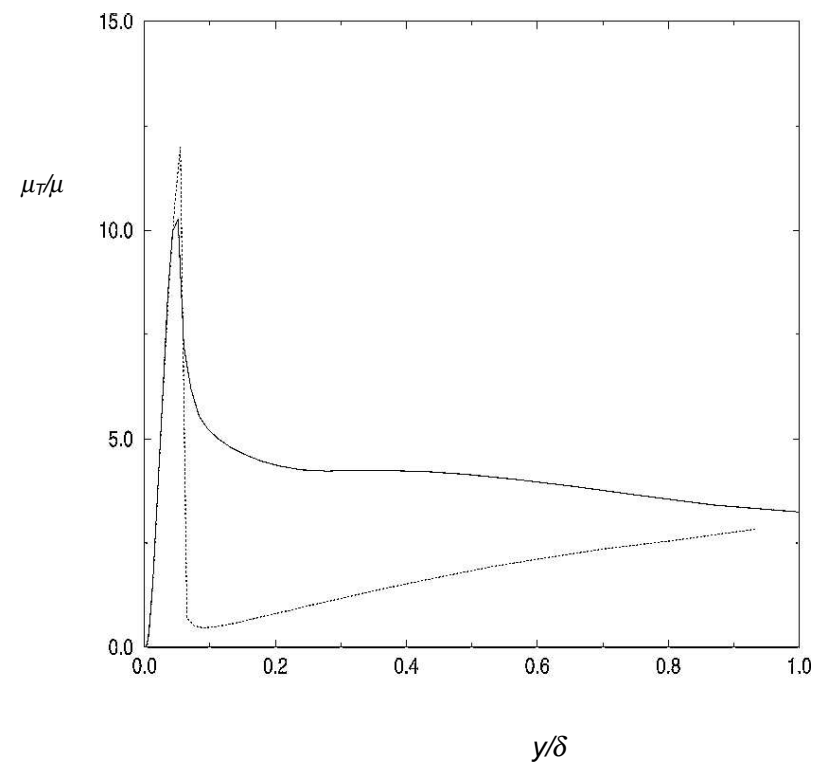

Fig. 5. Variation of $\mu / \mu_{T}$ with $y / \delta$.

( $\cdots \cdots \cdot$ Code (B), $\Delta=\Delta y$; - Code (A), $\left.\Delta=(\Delta x \Delta y \Delta z)^{1 / 3}\right)$ 


\section{v. CONCLUSIONS}

A relatively straightforward way to implement zonal $k$-l based LES/RANS approach has been presented. It is tested for periodic plane $\left(R e_{\tau}=1050\right)$ and ribbed channel flows $(R e=30,000)$. The interface for the differing models is explicitly specified. This is achieved via intrinsic wall distance based arguments. For the ribbed channel flow, wall distances are specified via a differential equation based technique. Using standard established LES and RANS model constants the zonal model is shown to reproduce a satisfactory law of the wall. The approach is implemented in both cell-vertex and cell-centered codes. For the differing codes, predictions are found within about $3 \%$ of each other. Various other channel flow sensitivity studies are performed. These show that, as with standard LES, predictions are most sensitive to filter size, first off wall grid node normal positions and temporal scheme order.

Sensitivities to established turbulence model constants, RANS/LES interface location and cross term modelling are found to be less. For a periodic ribbed channel flow the zonal LES heat transfer

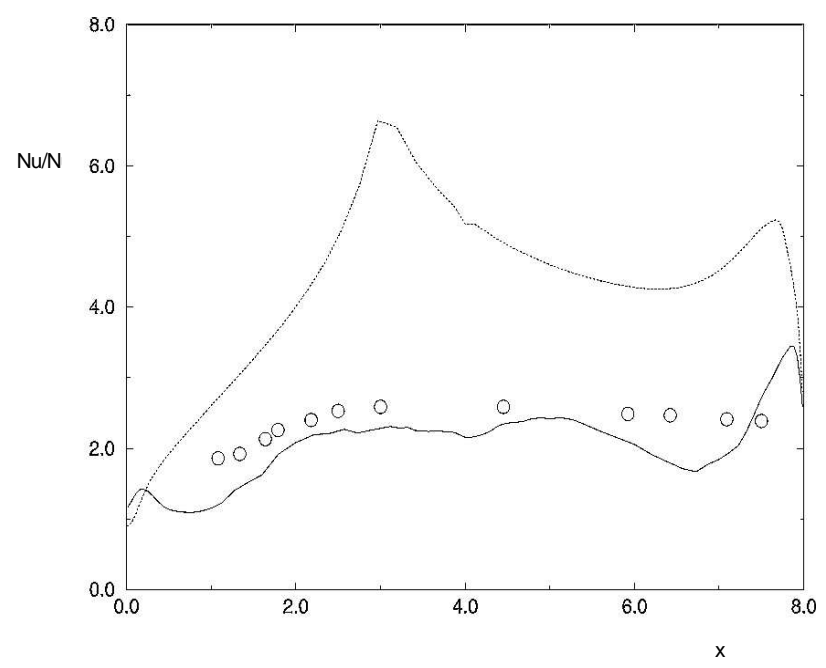

Fig. 6. Nusselt number predictions for ribbed channel flow (o Measurements; … RANS model; - Zonal LES/RANS model)

predictions are found to significantly more accurate than those for an established two equation RANS model. They are also marginally more accurate than those for an LES prediction using the same grid node number.

\section{ACKNOWLEDGEMENTS}

Engineering and Physical Sciences Research Council (EPSRC) funding under grant number GR/R34929/01 is gratefully acknowledged. The majority of this work was carried out at Chalmers.

\section{REFERENCES}

1. Tucker, P.G., 2001. Computation of unsteady internal flows, Kluwer Academic Publishers.

2. Rodi, W., Ferziger, J. H., Breuer, M. and Pourquie, M., 1997. Status of large eddy simulation: results of a workshop, Trans. of the ASME, 119, 248262.

3. Spalart, P.R., 1999. Strategies for turbulence modelling and simulations, Proc. 4th Int. Symp. on Engineering Turbulence Modelling and Measurements, Ajaccio, Corsica, France, 3-17.

4. Shur, M., Spalart, P.R., Strelets, M. and Travin, A., 1999. Detached-eddy simulation of an aerofoil at high angle of attack, Proc. 4th Int. Symp. on Engineering Turbulence Modelling and Measurements, Ajaccio, Corsica, France, 669-678.

5. Nikitin, N.V., Nicoud, F., Wasistho, B., Squires, K.D. and Spalart, P.R., 2000. An approach to wall modelling in large-eddy simulations, Physics of Fluids (Letters), 12, No. 7, July, 1629-1632.

6. Forsythe, R.F., Hoffmann, K.A. and Dietiker, J.F., 2000. Detached-Eddy simulation of a supersonic axisymmetric base flow with an unstructured solver, AIAA Paper 2000-2410.

7. Kumar, S. and Loth, E., 2001. Detached eddy simulations of an iced-airfoil. $39^{\text {th }}$ AIAA Aerospace Sciences Meeting and Exhibit, 8-11 January, 2001, Reno, NV, AIAA Paper 20010678.

8. Spalart, P.R. and Allmaras, S.R., 1994. A oneequation turbulence model for aerodynamic flows, La Rech. Aerospatiale, 1, 5-21.

9. Speziale C. G., 1987. On non-linear $k-l$ and $k-\varepsilon$ models of turbulence, J. of Fluid Mech., Vol. 178, pp. 459-475.

10. Arunajatesan, S., Sinha, N. and Menon, S., 2000. Towards hybrid RANS-LES computations of cavity flowfields. $38^{\text {th }}$ AIAA Aerospace Sciences Meeting and Exhibit, 10-13 January, Reno, NV, AIAA Paper 2000-0401.

11. Batten, P., Goldberg, U., Chakravarthy, S., 2000. Sub-grid turbulence modelling for unsteady flow with acoustic resonance. $38^{\text {th }}$ AIAA Aerospace 


\section{AIAA-2003-0082}

Sciences Meeting and Exhibit, 10-13 January, 2000, Reno, NV, AIAA Paper 2000-0473.

12. Peltier, L.J., Zajaczkowski, F.J. and Wyngaard, J.C., 2000. A hybrid RANS/LES approach to large-eddy simulation of high-Reynolds-number wall-bounded turbulence, Proceedings of ASME FEDSM'00: ASME 2000 Fluids Engineering Division Summer Meeting, June 11-15, 2000, Boston, Massachusetts, FEDSM2000-11177, 113-118.

13. Arunajatesan, S. and Sinha, N., 2001. Unified unsteady RANS-LES simulations of cavity flowfields. $39^{\text {th }}$ AIAA Aerospace Sciences Meeting and Exhibit, 8-11 January, Reno, NV, AIAA Paper 2001-0516.

14. Davidson, L., 2001. Hybrid LES-RANS: A combination of a one-equation SGS model and a k-omega model for predicting recirculating flows, Proc. ECCOMAS CFD Conference, Swansea, U.K.

15. Patankar, S.V., Liu, C.H. and Sparrow, E.M., 1977. Fully developed flow and heat transfer in ducts having streamwise periodic variations of cross-sectional area, ASME Journal of Heat Transfer, May, 99, 180-186.

16. Wolfshtein, M., 1969. The velocity and temperature distribution in one-dimensional flow with turbulence augmentation and pressure gradient, Int. J. Heat Mass Transfer, 12, 301-318.

17. Chen, H.C. and Patel, V.C, 1988. Near wall turbulence models for complex flows including separation, AIAA Journal, June, 26, No. 6, 641648.

18. Yoshizawa, A., 1993. Bridging between eddyviscosity-type and second order models using a two-scale DIA, $9^{\text {th }}$ Int. Symp. On Turbulent Shear Flow, Kyoto, 3, 23.1.1-23.1.6.

19. Fureby, C., 1999. Large eddy simulation of rearward-facing step flow, AIAA Journal, November, 37, No. 11, 1401-1411.

20. Sohankar, A. and Davidson, L., 2001. Effect of inclined vortex generators on heat transfer enhancement in a three-dimensional channel, Num. Heat Transfer, 39, No. 5, 433-448.

21. Chung, Y.M. and Tucker, P.G., 2001. Comparative study of numerical methods for unsteady incompressible flow calculations, submitted to J. of Computational Physics.

22. Rau, G., Cakan, M., Moeller, D. and Arts, T., 1998. The effect of periodic ribs on the local aerodynamic and heat transfer performance of a straight cooling channel, ASME J. of Turbomachinery, 120, April, 368-375.

23. Rollet-Miet, P., Luarence, D. and Ferziger, J., 1999. LES and RANS of turbulent flow in tube bundles, Int. J. of Heat and Fluid Flow, 20, 241254.

24. Spalart P.R., 2001. Young person's guide to detached-eddy simulation grids, Technical Report NASA/CR-2001-211032.

25. Piomelli, U., 1993. High Reynolds number calculations using the dynamic subgrid-scale stress model, Phys. Fluids, 5, 1484-1490.

26. Launder, B.E. and Sharma, B.I., 1974. Application of the energy-dissipation model of turbulence to the calculation of flow near a spinning disc, Letters in Heat and Mass Transfer, 1, 131-136. 